\title{
Towards Augmented Reality User Interfaces in 3D Media Production
}

\author{
Max Krichenbauer* Goshiro Yamamoto ${ }^{\dagger} \quad$ Takafumi Taketomi $^{\ddagger} \quad$ Christian Sandor $^{\S} \quad$ Hirokazu Kato $^{\mathbb{I}}$ \\ Interactive Media Design Laboratory \\ Nara Institute of Science and Technology
}
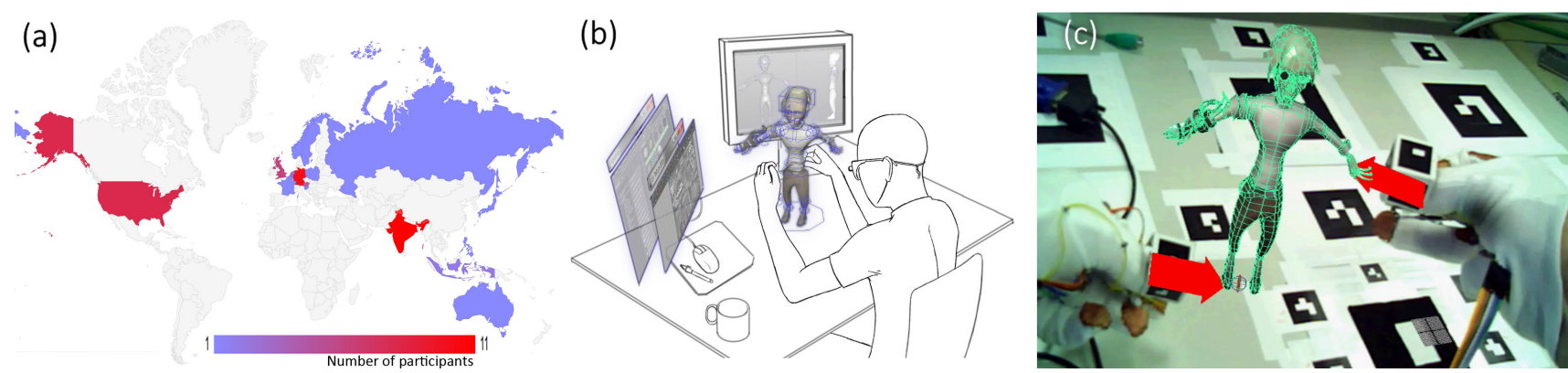

Figure 1: Our goal is to establish Augmented Reality as a user interface for professional media production. Our approach consists of three steps: We conducted an online survey with 3D media professionals from all around the world (a). Based on their responses, we derived requirements and designed a user interface (b). We then implemented a prototype (c) and evaluated it with media professionals in a pilot study.

\begin{abstract}
The idea of using Augmented Reality (AR) user interfaces (UIs) to create $3 \mathrm{D}$ media content, such as $3 \mathrm{D}$ models for movies and games has been repeatedly suggested over the last decade. Even though the concept is intuitively compelling and recent technological advances have made such an application increasingly feasible, very little progress has been made towards an actual real-world application of $\mathrm{AR}$ in professional media production. To this day, no immersive 3D UI has been commonly used by professionals for 3D computer graphics (CG) content creation.

In this paper, we are first to publish a requirements analysis for our target application in the professional domain. Based on a survey that we conducted with media professionals, the analysis of professional 3D CG software, and professional training tutorials, we identify these requirements and put them into the context of AR UIs. From these findings, we derive several interaction design principles that aim to address the challenges of real-world application of AR to the production pipeline. We implemented these in our own prototype system while receiving feedback from media professionals. The insights gained in the survey, requirements analysis, and user interface design are relevant for research and development aimed at creating production methods for $3 \mathrm{D}$ media production.
\end{abstract}

Keywords: Augmented Reality, Immersive Authoring

Index Terms: H.5.1 [Information Interfaces and Presentation]: Multimedia Information Systems-Artificial, Augmented, and Virtual Realities; I.3.6 [Computer Graphics]: Methodology and Techniques-Interaction techniques

\footnotetext{
*e-mail: max-k@is.naist.jp

†e-mail:goshiro@is.naist.jp

†e-mail:takafumi-t@is.naist.jp

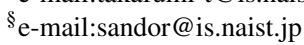

Ile-mail:kato@is.naist.jp
}

\section{INTRODUCTION}

Even before the advent of AR, the idea of creating an immersive 3D modeling application for creating 3D content was proposed by Clark [7]. However, even though AR has been shown to be excellent for 3D tasks thanks to correct spatial alignment [24, 26], there is, to our knowledge, no fully functioning immersive 3D UI available for professional 3D content creation, including film, TV, or games. A number of AR CAD applications were created and tested by researchers, but none of them was based on a requirements analysis of professional 3D design work or was further developed for professional production. In order to bridge the gap between researchers and studios, we analyzed the requirements of digital content creation and derived a UI design from them.

We limit our work to the $3 \mathrm{D}$ content creation part of the production: modeling, rigging, animation, simulation, and lighting/rendering. We do not consider $2 \mathrm{D}$ elements of the production pipeline such as compositing or color-grading, since this work is naturally two-dimensional and thus differs greatly from the scrutinized tasks. We also do not consider authoring of AR experiences: this paper is about using AR as a means of producing 3D content, not about using $3 \mathrm{D}$ content to create AR applications.

In order to provide a scientifically sound analysis of professional 3D design work, we have included several sources of information. Primarily, we performed a survey with 3D professionals worldwide (section 3). We also analyzed the UIs of existing professional 3D content creation software. Furthermore, to gain more insight into the common use of the software, we reviewed tutorial videos aimed at professional 3D design training, which cover the use of the software as well as production principles. Based on these sources, we deduced requirements for UIs for $3 \mathrm{D}$ design, and discuss a set of UI concepts that aim to address these issues.

Contribution: Our main contribution is a thorough requirements analysis of UIs for professional 3D media design. Our findings will allow more application oriented research and development in this area. Furthermore, we suggest a number of interface designs addressing the challenges, which we identified. Taking feedback from media professonals, we combined these designs into a integrated work environment. Our findings can help advance the search for universal UI principles for AR 3D modeling. 


\section{Related Work}

Previous publications related to AR UIs for professional 3D media creations can be categorized as follows: reviews that give an overview and analysis of previous attempts at create AR UIs; authoring tools that allow a limited form of content creation by assembling predefined objects; AR UIs for engineering CAD purposes; immersive 3D modelers; AR related UIs targeted at professional CG software and procedures; and novel UIs for 3D design that are not AR or Virtual Reality (VR) related. In the following, we discuss the most prominent publications from each category.

Reviews of real-world applications of AR. It has been a common problem of research efforts to bridge the gap to commercial application. Fite-Georgel [11] surveyed a large number of systems on their applicability and successful adoption for industrial applications such as manufacturing and construction. Our focus is on virtual 3D media content creation, which is more of an artistic discipline than precise engineering. LaViola and Keefe [19] give an overview of past and recent 3D UIs for art and design purposes, without having a specific viewpoint of media production.

AR authoring tools. Another area of vibrant research are immersive authoring systems. In these systems, the user assembles a world out of provided predefined content by interactive placement and changing specific attributes such as texture. FingARtips [4] offers glove-based within-arms-reach object manipulation in an AR tabletop environment, aimed at urban planning. Thus the interaction is limited to placing houses and streets. Two recent systems were presented at the IEEE 3D UIs Contest: the DIY World Builder [27] and the Wonderland Builder [3], targeting level creation for games and creating fantasy worlds. While there is some requirement in professional 3D design to incorporate previously prepared or acquired objects, UIs that are limited to content assembly are not sufficient for professional design.

AR UIs for engineering. Engineering also has a high demand for creation of virtual 3D models, and a number of AR based UIs were developed. These systems are commonly called ARCAD and focus on precision and physical correctness instead of artistic expression. Girbacia [13] developed an ARCAD system to cooperate with available legacy 3D CAD software, offering only 3 features (creating a primitive object, extruding 2D closed profiles and revolving a $2 \mathrm{D}$ profile around an axis). Gao [12] proposed a semiimmersive virtual environment for solid modeling using voice commands to extend the UI. Shen et al. [23] developed a system that combines an AR UI with remote collaboration for several engineers, exploring principles how to protect technical correctness in a collaborative set-up.

Immersive 3D modelers. Using AR as a UI for artistic 3D modeling, animation, etc. as we intend it has been proposed by several authors, however without concerning the professional application. 3Dm [5] was one of the first systems that explored the design space of immersive modeling in a VR setting. The THRED system [22] offers bi-manual modeling using 3D tracked "bats". Similarly, JD$\mathrm{CAD}+[14]$ is a non-immersive modeling and animation system that uses a 6 degrees of freedom (DOF) tracked "bat" to create content for virtual environments. 3darmodeler [9] is a stand-alone AR based modeler directed toward amateurs. Kim et al. [16] developed immersive VR modeling system that is based on 5 hand gestures for basic Subdivision-Surface modeling. The outdoor AR system Tinmith [20] was also used for creating 3D models in a very limited way. Construct3D [15] is a modeling system which aims for mathematical education. ICOME [21] explored remote collaboration in immersive modeling environments. None of these systems concerns itself with professional application in media production.

AR UIs for CG software. Only a small number of publications concerned itself with the possibilities and demands of professional application. ARpm[10] is an AR front-end to Autodesk ${ }^{\circledR} 3 D$ Studio Max ${ }^{\circledR}$, which functions by taking screenshots of the software and sending Windows system calls to control it which greatly limits performance and usability. Takala et al. [25] implemented a semi-immersive 3D UI for the Blender modeling software using a Playstation3 and a number of PlayStation Move controllers. Their aim was to create an inexpensive set-up. Most interestingly, they tested their prototype with 7 professional 3D artists. However, their evaluation only concerned the use of the prototype, whether it was "fun" or whether they experienced "fatigue" during their 25 minute use time. Barakonyi published a system that targets character animation exclusively by using a physical puppet [2]. The concept of tracking a physical rig — originally developed by Knep et al. [18] - was improved by applying AR technology. Such an approach is logically limited to a very narrow application: only animation of virtual content which can be represented by a physical puppet (no fluids, hair or muscle rigs) and no possibility to play back recorded animation, forcing the animator to work "straight-ahead" which is uncommon in professional animation. Most importantly, none of these publications includes an analysis of requirements for professional application. The only commercially available AR application directed toward professional 3D CG content creation known to us are the ARplugins. However, these plug-ins to 3D content creation software merely offer visualization of the content. There is no way to create or edit models in the AR environment.

Non-AR novel 3D user input devices. Another area of research and development are novel user input devices for 3D design. These commonly try to offer more DOF while still using a normal monitor set-up. Autodesk Inc. ${ }^{\circledR}$ offers a Plugin for their Maya ${ }^{\circledR}$ modeling software that uses a Leap Motion controller to track the users hands, allowing some interaction with the virtual content [1]. The Didjiglove [8] is a glove-based user input system to control animations in professional 3D software by using it as an advanced programming interface. Similar to the previously mentioned concept of employing a physical puppet, QUMARION [6] is a currently commercialized version without the AR overlay proposed by Barakonyi.

\section{SURVEY ON 3D DESIGN WORK}

We performed a survey with $3 \mathrm{D}$ media professionals in order to gather a solid empirical base of information. In the following, we give information on how we performed the survey and present the most interesting results, including questions related to the work load and health issues, common forms of collaboration in the studio environment, amount of functionality habitually used by artists, complexity of the content worked with, as well as expectations on future UIs. The complete survey results are provided as supplementary material.

We used an online survey platform in order to reach professionals from around the globe. The questions included a selfassessment of professionalism and skills in order to get an impression of the participant. Where the self-assessment did not suit our requirements for industry professionals we did not include the data. We distributed the survey via various channels including our own personal contact network, 3D art forums and freelancer recruiting websites. In order to ensure authenticity we asked the name and company or portfolio website of all participants. We did so at the end of the survey after all answers had been completed and ensured the participant that their names would not be published in order to avoid social desirability bias. Answers of participants who could not be identified were then removed from the sample. We did not contact each individual artist, but the timing of responses after informing different groups of people about the survey gave us confidence that the person did in fact take the survey. The final sample consisted of 54 participants from 17 countries (Figure 1(a)).

We asked a number of questions regarding the artists work environment and daily work context. When asked for their average daily work hours, the average time stated was 6.87 hours (standard deviation (SD) 2.27 hours ), where the common duration of one 


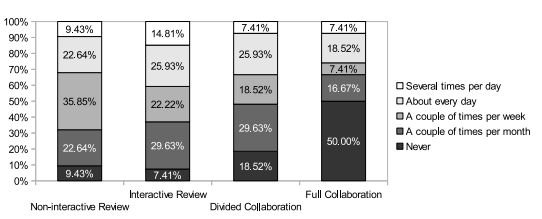

(a)

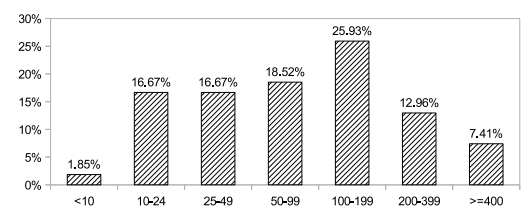

(d)

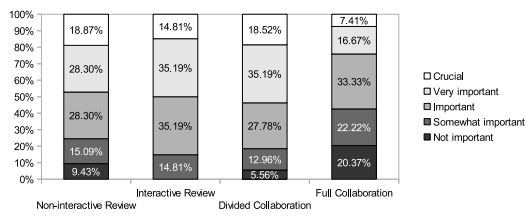

(b)

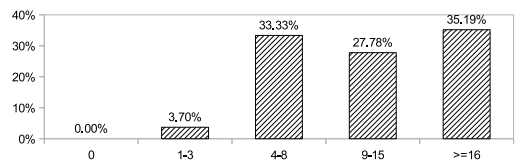

(e)

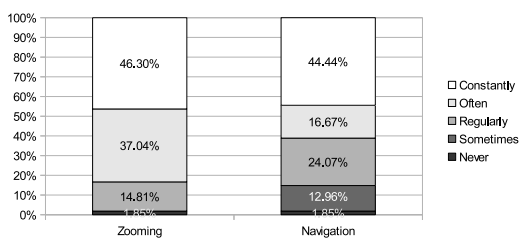

(c)

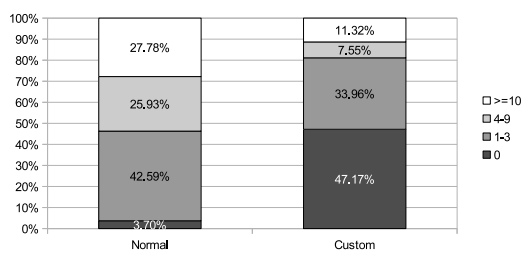

(f)

Figure 2: (a) Frequency of various types of collaboration. (b) Perceived importance of various types of collaboration. (c) "Please estimate the complexity/size of content you are working with." (d) "Estimate how many different tools and functions of 3D CG software you regularly use." (e) "Estimate how many keyboard hot-keys for your 3D software you use regularly." (f) Estimation and classification of additional scripts or plug-ins used with 3D software; (normal) means publicly available, (custom) means developed in-house or ordered exclusively.

work session until taking a break was on average 3.02 hours (SD 2.25 hours). When asked for their current main input device, all artists expressed working with a $2 \mathrm{D}$ device, $75.47 \%$ with a mouse and $24.53 \%$ with a pen tablet. Not a single participant stated using an alternative or 3D user input device for work. More than $16.67 \%$ of artists reported that they previously tried 3D user input devices, either haptic 3D input device $(3.70 \%)$, 3D mouse $(11.11 \%)$, or a 3D spheric mouse $(5.56 \%) .1 .85 \%$ stated to have used a glove based input device. When asked how their arms, hands and wrists feel after several days of working with 3D software, $46.67 \%$ stated "normal", $46.67 \%$ expressed that their arms felt "tired" and $6.67 \%$ even stated that their hands "hurt". However, when asked whether they perform some kind of countermeasures to deal with work related problems in their arms, wrists or hands, a total of $31.48 \%$ stated that they employ some kind of counter measure (special hand-gear: $1.85 \%$; special exercises: $29.63 \%$; take medication: $1.85 \%$; seeing a physician or health expert: $3.70 \%$ ). We further found that $38.89 \%$ of the participants are missing a third dimension in their UI (monitor: $18.52 \%$, mouse: $35.19 \%$ ). However, $27.78 \%$ also expressed explicitly positive opinions about 2D UIs.

To get an impression of collaboration in the studio environment, we divided the various types of collaboration into four categories. Non-interactive review was defined to be the process of presenting the content without being able to edit it at the same time. Interactive review similarly was described as receiving feedback while simultaneously being able to edit the content. Divided collaboration was defined as the process of multiple artists working on the same content by means of dividing it either by separation or taking turns. Full collaboration finally defined working together on the same content at the same time. We asked the participants both how regularly they performed each type of collaboration and how important they consider it - whether based on experience or expectation-for their professional work. The non-interactive review and interactive review were considered the most common and important form of collaboration, but even full collaboration, while relatively rare, was considered important by the majority of artists. (Figure 2(a), 2(b)).

One set of questions related to the way that artists used their professional tools daily. We asked them how many of the functions that their 3D software offers they actually use regularly. We discovered that it is common for 3D artist to use up to a hundred functions (Figure 2(d)). Furthermore, we asked how many keyboard hot-keys they habitually used to increase their efficiency. We found that using 16 or more hot-keys was not a rare exception, whereas using less than or 3 hot-keys is uncommon (Figure 2(e)). When asked whether they use scripts or plug-ins for their software that were not part of the original set of functionality, only $3.70 \%$ stated not to require any additional functionality, and many artists use more than just a few additional scripts or plug-ins (Figure 2(f)). Similarly, $52.83 \%$ of the participants in our survey stated to use custom scripts or plug-ins that where developed at their own company, or were ordered to be created exclusively for their own requirements. $77.78 \%$ stated that they believe custom scripts and plug-ins will always be a requirement in order to adjust the software to production requirements.

We asked about the use of translational navigation and zooming/scaling during work on a 5-bin Likert scale, ranging from "constantly" to "never" (Figure 2(c)).

Finally, we presented artists with a series of novel user input devices, as they were previously proposed by scientists: AR overlay applied to a haptic 3D input device, 3D mouse, gloves, and a mannequin, as well as voice input. We asked the participants to rate their agreement to common arguments regarding the advantages and disadvantages of these systems in form of a Likert Scale. It became apparent that media professionals agree considerably more with the shortcomings of novel user input devices than with their expected merits. We also asked them to rate the usefulness of the presented devices on a Likert scale from 1 ("very useless") to 7 ("very useful"), comparing them to each other as well as to the traditional mouse as a user input device for 3D content creation. Strikingly, all 3D input devices scored significantly lower (between 4.50 and 4.25$)$ than the traditional 2D mouse (6.59). Voice input scored even lower than the 3D UI devices (3.30).

\section{Derived Requirements}

In this section we present information on specific requirements for the professional 3D content creation workflow. These are: an er- 
gonomic design that can be used comfortably for a long period of time, support for collaboration among artists and supervisors, a high amount of features, support for navigating the content, support for $2 \mathrm{D}$ and alphanumeric operations, and an increase in productivity over the traditional 2D UI. The subsections are in no particular order, as all points are independent requirements.

Ergonomic design. As our survey has shown, 3D artists tend to work for several hours before taking a break and spend a lot of time working with their software every day. Even with the quite comfortable and sophisticated traditional UIs, this becomes an issue for the artists health and well-being. It is therefore important to provide UIs that are comfortable to use over an extended time without strain or tiredness. Previous publications only provided quick user tests and thus never discussed problems encountered during extended use, which are vital for professional application. For example, some researchers suggested systems where the users interact while standing $[15,20]$. While this may be comfortable or even healthy for a short time period, it is unlikely to be adopted for extended use. Furthermore, the device used for viewing the AR must be considered. Using a tablet or other mobile device requires the artists to move it around whenever the viewing position must be changed. As our survey has shown, this is basically constantly the case. Hence, a head-mounted display (HMD) is more appropriate.

Collaboration support. Since professional production is not an individual effort of a single artist, some kind of collaboration is obviously necessary. According to our survey and own experience, many types of collaboration are commonly performed and necessary for producing high-quality results. In the most basic way, this consists of showing the content to another person while the user is able to perform changes simultaneously. The traditional use case being that the supervisor or a colleague visits the artist at his work desk, and comments on the current state of the work. Traditional 2D UIs support this naturally because the monitor screen is visible to everyone, and via pointing and talking, feedback can be communicated. Novel UIs however must be designed with this requirement in mind. This requires some way of bidirectional information transfer: other people than the artists must be able to perceive the virtual content, and must be able to communicate with the artists about the content. AR has an inherent advantage over VR in this respect because all users in a shared augmented environment can see each other without need for virtual representation. Hence, pointing at specific parts of the content while talking with each other directly is possible. For VR and remote collaboration, similar communication methods must be provided. However, reviews are not the only type of collaboration used or wished for by artists. While traditional PC UIs are inherently single-user (even when several mouses and keyboards are connected, the single focus makes real collaboration impossible), some types of simultaneous work through quick alteration, file versioning or separation of tasks are also common or at least estimated to be useful by most artists. Novel systems can break with the traditional single-user paradigm and offer real time multi-user collaboration.

High amount of features. While in industrial applications correctness is usually the greatest challenge [23], media production challenges us with an intense amount of functionality. Because there is a huge pressure to produce more and more stunning expressive imagery, the accumulated feature set by far exceeds any existing AR or VR application. These functions include tools like translation or rotation, actions like extruding or collapsing a polygon, operational modes like interacting with single vertices or complex group hierarchies of objects, mathematical operations like boolean difference of two bodies, computer graphics features like different types of light sources, materials or cameras, or support for industry standards like automation APIs, scripting or file types. As our survey illustrates, these features are actively used by the artists, and that fast input methods like hot-keys are a necessity. Even more problematic: since most artists extend their feature set with additional scripts and plug-ins (sometimes even individually customized) it is impossible to anticipate the required features. While the technical challenge seems daunting, it is actually easily solved: instead of trying to implement all required functionality, one can easily build on existing software either by integrating open-source code or by offering the UI in the form of a plug-in for existing software. The real challenge lies in the UI design: no previous system known to us attempted to provide access to a similar amount of functionality while still being intuitive. Novel approaches are required to organize complexity in $3 \mathrm{D}$ UIs.

Fast and intuitive navigation. Another important factor that stems from the complexity of the content processed in professional production is the need to navigate through the content. In this context we use the term navigation in its traditional meaning of changing the relative position of the users viewpoint and the virtual environment or content without altering the content (editing). In immersive VR environments, the term and reasoning is intuitive to understand because the user has a sense of "moving" through the environment. AR applications typically don't incorporate any navigation other than physical (by moving the camera or moving a fiducial marker) because the user can still see the real environment, so there would be no sense of personal motion. However, in digital media production, the content is too big and complex for physical navigation to be feasible. Some scenes may incorporate whole cities, where the artist is working on figures in individual streets while trying to keep an overview of the complete scene. Our survey confirms this: no one stated that they can perform their work without any form of zooming or navigation - on the contrary, most artists navigate their content often or constantly during work. Therefore, applications have to implement intuitive and quick user navigation.

Support for 2D and alphanumeric operations. 3D design consists of a greater number of sub-tasks, not all of which are 3-dimensional in themselves. Some tasks are inherently 2dimensional in nature, even though they relate to 3D CG. An example for this is texture mapping, where the "UV" coordinates of vertices of polygonal models must be laid out on a 2D map. Here, the $3 \mathrm{D}$ artist tries to achieve a balance between distortions in the texture, seams in texture mapping, limited space on the 2D map and ease-of-use for texturing artists. Another problem that is difficult to realize in 3D UIs is the need for alphanumerical input. This is often required in 3D design in the form of file names or inputting exact parameter numbers. Moreover, in rigging, it is often required to perform some minor scripting on the 3D content, such as defining a relationship between several objects to implement some mechanics. One way to offer alphanumeric input is via voice input over a microphone, as proposed by Girbacia et al. [13]. However, a talking-out-loud approach is not very suited for a shared workplace environment. Throat microphones might alleviate the problem at the expense of increased cost, decreased comfort and a loss in reliability. Our survey shows that $21.2 \%$ of the participating artists disagree to any usefulness of voice input, and only $36.4 \%$ see it as a possible replacement for traditional mouse-and-keyboard UIs.

Increase in productivity. Surprisingly, one of the greatest challenges is to design a UI that actually matches or even outperforms traditional 2D UIs in terms of efficiency. It is easy to believe that a 3D UI will in itself increase ease of use and user performance thanks to spatial understanding. In our user tests however, we had to discover that this is not the case: spatial understanding is limited, even when stereo vision HMDs are used, and the limited advantage of some depth perception is hardly enough to encourage switching systems. As our survey shows, artists are open to alternative 3D input devices, but critical in their adoption. They have developed skills to work with 2D UIs with high speed and precision. Our survey has shown the limited hope of artists that alternative UI concepts can live up to working with a traditional 2D mouse. 

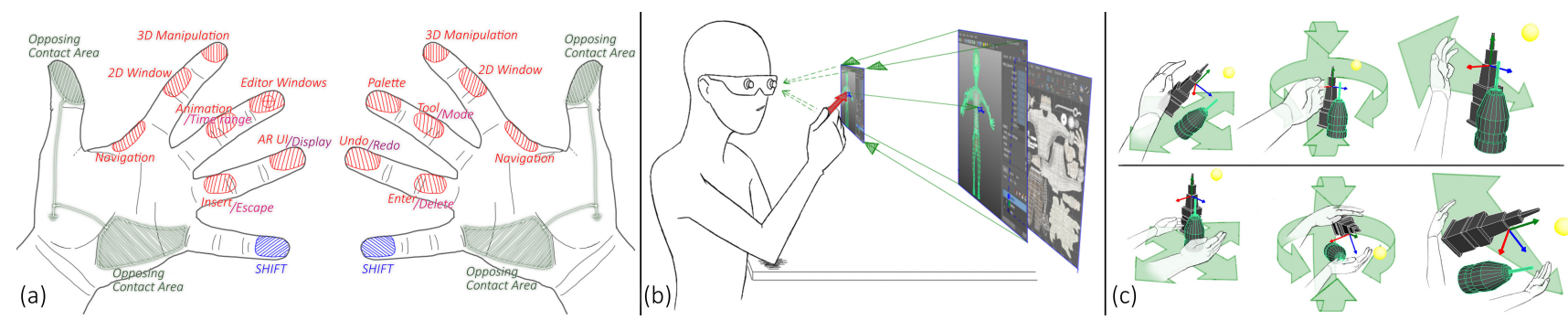

Figure 3: UI design concepts. (a) Mapping of functions to the hand. (b) 2D window interaction. Inactive windows face the user beyond arms reach. Ray casting from the dominant eye over the cursor is used for interaction. When a window becomes active, it is moved forward, changing its size accordingly so that the optical impression will not change. (c) Navigation concept: the world origin is transformed to give the illusion of positioning the objects without editing. Top: Single handed navigation, "zooming" with the Shift key (right). Bottom: Bi-manual navigation.

\section{User INTERface Design}

Based on this set of requirements, we developed a concept for a 3D UI to meet the requirements for professional 3D design work. Figure $1 \mathrm{~b}$ shows an illustration of the suggested UI. In our system, the artist is comfortably seated at his work desk, wearing a stereo video-see-through HMD. The artists hands are tracked to allow direct manipulation within arms reach. An AR system is used over VR to allow interaction with everyday objects (eg. a coffee cup) and other people (important for collaboration). The 3D AR UI should be implemented as a plug-in or extension of the respective software package that the artist uses. This allows seamless transition between traditional and AR UI, and automatically provides all the features and integration requirements of the production.

Ergonomic direct hand interaction. We advocate bi-manual direct hand interaction within arms reach as main input vector, as it is the most natural and versatile way of interaction. The user can rest the elbows or forearms on the table while working to reduce the strain during extended work. The thumbs of both hands are the frame of reference for pinch-based interaction, which allows a natural "grabbing" interaction metaphor. The main interaction functionality (editing 3D content, using 2D elements and navigation of the scene) is implemented on both hands in the same way, allowing simultaneous bi-manual interaction. Glove based systems have been shown to support a great number of buttons comfortably [8], which supports our requirement to offer a high amount of functionality. In our UI design, we implement 8 pinch-buttons per hand, with two complementary contact areas: the tip of the thumb and the palm of the hand. The tip of the little finger assumes the function of a Shift key, temporarily altering the assigned functions of the other buttons. Thus, we offer an effective number of 14 keys per hand. Figure 3(a) shows the mapping of functions on the hands.

Navigation. When the "navigation" button on top of the hand is pressed, the whole 3D content (ie. world origin) will change its position in the work area as if being grabbed by the user's hand, in 6DOF (translation and rotation), realizing the "grabbing-theair" concept. This technique is intuitive not only in immersive virtual environments - where it gives the impression of pulling oneself through the environment-but also when working with a single virtual object in an AR environment, where it feels similar to grabbing and positioning the object in the work space. Thus, it automatically realizes a "frame-of-reference" concept for hand interaction: the user holds the object (in fact: the whole virtual scene) with the non-dominant hand while working on it with the dominant hand. Zooming is performed with the Shift button, or by using two hands simultaneously (Figure 3c).

Collaboration. Novel 3D UIs can support collaboration on two levels: interactive reviews and full real-time collaboration. In the most simple case, it should be possible to perform interactive reviews by displaying the virtual content on the computer monitor by cloning the video stream of the artists HMD, or by employing additional cameras and displays. In order to allow full collaboration, simultaneous interaction is required which is harder to achieve since it conflicts with the requirement for simple navigation: if several artists share the same global coordinate system, they would interfere with each others work when they change it. Kiyokawa's proposed separation between personal and public spaces [17] can be applied here, offering separated coordinate systems to every user as well as a global coordinate system for exchange.

Menus offering a high amount of operations. As shown earlier, the range of tools and operations provided by professional 3D software is huge. We propose a palette way of organizing functionality that uses the natural range of freedom of the hand interaction. The visual feedback was found to be most convenient when 2D, facing the user, and using the third DOF of hand motion (distance) to navigate several layers of tools and options.

2D editor windows. Apart from the obvious advantage of AR that the user is still able to use traditional UIs without having to switch, we also developed a 3D approach to 2D interactions. All 2D window elements in the AR view face the user at all times. When inactive, they reside at a background plane beyond arms reach so they don't interfere with the interaction. The user can point at these windows by hovering the thumb cursor over them, as seen by the dominant eye (i.e. by casting a ray from the dominant eye over the cursor onto the window). When a window gains focus, it is brought forward towards the cursor to eliminate the binocular disparity. When the window comes forward or returns to the background, it is re-scaled accordingly to keep its apparent 2D size to avoid irritating the user (Figure 3(b)).

Efficient 9DOF manipulation. Traditional 2D UIs can only offer two DOF at a time and even 3D user input devices usually only offer 6DOF (more DOF may be theoretically available, but the user has to switch interaction and is thus is unable to control them simultaneously). Our bimanual 3DUI can offer object manipulation with all 9DOF simultaneously in a intuitive and efficient way: one hand controls translation and rotation, the relative position of the second hand controls scaling in three dimensions. It is intuitive to understand: one hand grabs and moves an object, and the second hand stretches or squashes it.

Improved animation time control. Time control for animation is usually implemented in the form of a 1D slider operated with the mouse. We implement an animation key, which allows "flipping" through the animation with a simple wave of the nondominant hand, similar to traditional hand-drawn animation where the artist would flip though a number of frames. Since the dominant hand can still be used to manipulate objects simultaneously, the artist can create coarse animations very fast (Figure 4). 


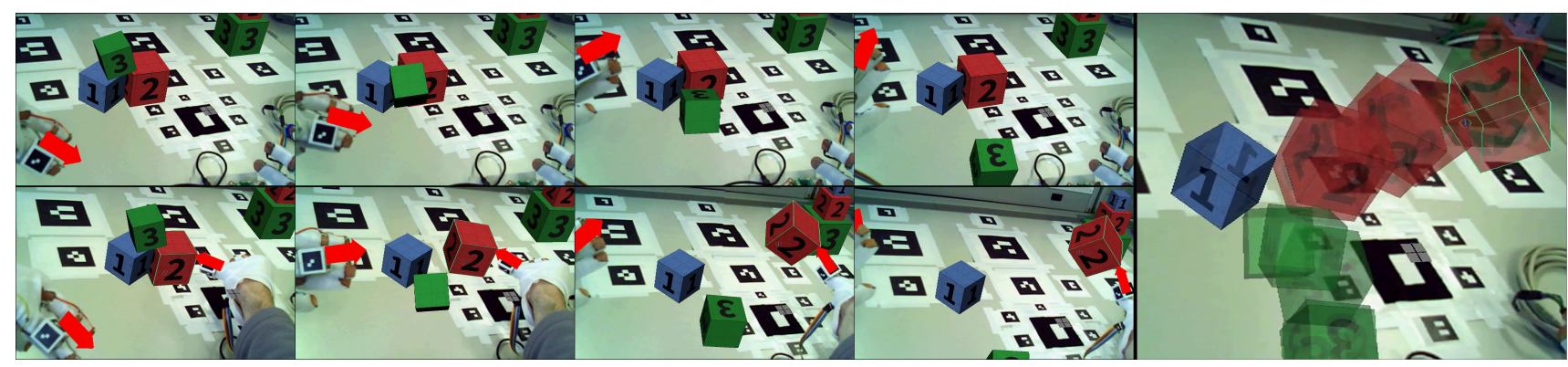

Figure 4: The user's view through the HMD while animating. Top row: The left hand controls the time. Bottom row: The right hand can be used to simultaneously manipulate objects, creating rough animation keyframes very fast. Right: Resulting animation.

\section{Prototype}

We implemented our recommendations on 3D AR UIs in a prototype (Figure $1(\mathrm{c})$ ) in the form of a plug-in for Autodesk ${ }^{\circledR}$ Maya ${ }^{\circledR}$. We used thin cotton gloves as user input devices by sewing conductive materials on them. Tracking of cameras and hands was achieved with ARToolKit. Throughout the development of the prototype, we stayed in contact with media professionals who agreed to test and comment on the system. This gave us valuable insights that helped advance both theoretical understanding as well as practical implementation.

\section{CONCLUSION}

In this paper we presented information on applying AR UIs to digital media content creation. We showed the challenges and problems for such applications and proposed several solutions which we have tested in form of a prototype.

As future work, we plan to perform a mature user study with media professionals.

\section{REFERENCES}

[1] Autodesk Inc. Leap Motion Plug-in for Autodesk ${ }^{\circledR}$ Maya ${ }^{\circledR} 2014$. http://area.autodesk.com/MayaLeapPlugin/, 2014.

[2] I. Barakonyi and D. Schmalstieg. Augmented reality in the character animation pipeline. In ACM SIGGRAPH 2006 Sketches, page 75. ACM, 2006.

[3] C. Barot, K. Carpentier, M. Collet, A. Cuella-Martin, V. Lanquepin, M. Muller, E. Pasquier, L. Picavet, A. Van Ceulen, and K. Wagrez. The wonderland builder: Using storytelling to guide dream-like interaction. In 3D User Interfaces (3DUI), 2013 IEEE Symposium on, pages 201-202. IEEE, 2013.

[4] V. Buchmann, S. Violich, M. Billinghurst, and A. Cockburn. Fingartips: gesture based direct manipulation in augmented reality. In Proceedings of the 2nd international conference on Computer graphics and interactive techniques in Australasia and South East Asia, pages 212-221. ACM, 2004.

[5] J. Butterworth, A. Davidson, S. Hench, and M. T. Olano. 3dm: A three dimensional modeler using a head-mounted display. In Proceedings of the 1992 symposium on Interactive $3 D$ graphics.

[6] CELSYS Inc. QUMARION - Humanoid input device. http://www.clip-studio.com/quma/en/, 2014.

[7] J. H. Clark. Designing surfaces in 3-d. Communications of the ACM, 19(8):454-460, 1976.

[8] L. Dipietro, A. M. Sabatini, and P. Dario. A survey of glove-based systems and their applications. Systems, Man, and Cybernetics, Part C: Applications and Reviews, IEEE Transactions on, 38(4).

[9] T. V. Do and J.-W. Lee. 3darmodeler: a 3d modeling system in augmented reality environment. International Journal of Computer Systems Science and Engineering, 4(2):145-154, 2008.

[10] P. Fiala and N. Adamo-Villani. ARpm: an augmented reality interface for polygonal modeling. In Mixed and Augmented Reality, 2005. Pro- ceedings. Fourth IEEE and ACM International Symposium on, pages 196-197. IEEE, 2005.

[11] P. Fite-Georgel. Is there a reality in industrial augmented reality? In Mixed and Augmented Reality (ISMAR), 2011 10th IEEE International Symposium on, pages 201-210. IEEE, 2011.

[12] S. Gao, H. Wan, and Q. Peng. An approach to solid modeling in a semi-immersive virtual environment. Computers \& Graphics, 24(2):191-202, 2000.

[13] F. Girbacia. An approach to an augmented reality interface for computer aided design. Annals of DAAAM \& Proceedings, 2010.

[14] M. Green and S. Halliday. A geometric modeling and animation system for virtual reality. Communications of the ACM, 39(5).

[15] H. Kaufmann. Construct3d: an augmented reality application for mathematics and geometry education. In Proceedings of the tenth ACM international conference on Multimedia.

[16] H. Kim, G. Albuquerque, S. Havemann, and D. W. Fellner. Tangible 3d: Hand gesture interaction for immersive $3 \mathrm{~d}$ modeling. In IPT/EGVE, pages 191-199, 2005.

[17] K. Kiyokawa. 3d collaboration using mixed reality technology. In Proc. of the First International Symposium on Universal Communication, pages 100-109, 2007.

[18] B. Knep, C. Hayes, R. Sayre, and T. Williams. Dinosaur input device. In Proceedings of the SIGCHI conference on Human factors in computing systems, pages 304-309. ACM Press/Addison-Wesley Publishing Co., 1995.

[19] J. J. LaViola and D. F. Keefe. 3d spatial interaction: applications for art, design, and science. In ACM SIGGRAPH 2011 Courses, page 1. ACM, 2011.

[20] W. Piekarski. 3d modeling with the tinmith mobile outdoor augmented reality system. Computer Graphics and Applications, IEEE, 26(1):14-17, 2006.

[21] C. Raymaekers, T. De Weyer, K. Coninx, F. Van Reeth, and E. Flerackers. Icome: an immersive collaborative $3 \mathrm{~d}$ object modelling environment. Virtual Reality, 4(4):265-274, 1999.

[22] C. Shaw and M. Green. Two-handed polygonal surface design. In Proceedings of the 7th annual ACM symposium on User interface software and technology, pages 205-212. ACM, 1994.

[23] Y. Shen, S. Ong, and A. Nee. Augmented reality for collaborative product design and development. Design Studies, 31(2):118-145, 2010.

[24] R. Shepard and J. Metzler. Mental rotation of three-dimensional objects. Science, 1971

[25] T. M. Takala, M. Makarainen, and P. Hamalainen. Immersive 3d modeling with blender and off-the-shelf hardware. In $3 D$ User Interfaces (3DUI), 2013 IEEE Symposium on, pages 191-192. IEEE, 2013.

[26] M. Tonnis, C. Sandor, C. Lange, and H. Bubb. Experimental evaluation of an augmented reality visualization for directing a car driver's attention. In Proceedings of the 4th IEEE/ACM International Symposium on Mixed and Augmented Reality, pages 56-59. IEEE Computer Society, 2005.

[27] J. Wang, O. Leach, and R. W. Lindeman. DIY World Builder: an immersive level-editing system. In 3D User Interfaces (3DUI), 2013 IEEE Symposium on, pages 195-196. IEEE, 2013. 